\title{
Construção de conjuntos de imagens emocionais associadas a partir do International Affective Picture System
}

\author{
Creation of sets of associated emotionally-evocative pictures using the International Affective \\ Picture System
}

\begin{abstract}
Luciano Haussen Pinto', Luiza Ramos Feijón ${ }^{2}$, Lilian Milnitsky Stein ${ }^{3}$
${ }^{1}$ Mestre. Professor, Curso de Psicologia, Centro Universitário Franciscano (UNIFRA), Santa Maria, RS. ${ }^{2}$ Psicóloga. Mestranda em Cognição Humana, Programa de PósGraduação, Pontifícia Universidade Católica do Rio Grande do Sul (PUCRS), Porto Alegre, RS. Bolsista, Coordenação de Aperfeiçoamento de Pessoal de Nível Superior (CAPES). ${ }^{3}$ Doutora. Professora titular, Pós-Graduação em Psicologia, PUCRS. Bolsista, Produtividade em Pesquisa, Conselho Nacional de Desenvolvimento Científico e Tecnológico (CNPq).
\end{abstract}

Suporte financeiro: Conselho Nacional de Desenvolvimento Científico e Tecnológico (CNPq).

\section{Resumo}

Introdução: A forma como estímulos emocionais são processados e se relacionam com a cognição possui diversas implicações no campo da saúde mental. Há, portanto, a necessidade de instrumentos efetivos que viabilizem a mensuração da emoção.

Objetivos: Criar, a partir do International Affective Picture System (IAPS), 20 conjuntos de fotos associadas temática, visual e emocionalmente, e eleger a foto mais representativa de seu respectivo conjunto; investigar a correlação entre os fatores de associação (emocional, temática e visual); e verificar a correlação de emocionalidade dos 20 conjuntos com os dados da versão brasileira do IAPS.

Método: Um total de 353 participantes avaliaram e julgaram o quão associadas entre si eram as fotos de cada conjunto e qual era a foto mais representativa de cada conjunto.

Resultados: Dezoito dos 20 conjuntos demonstraram alta associação nos três fatores investigados. Em todos os conjuntos, foram obtidas hierarquias de representatividade. Houve significativa correlação positiva entre os fatores de associação temática e visual (coeficiente de correlação de Spearman, rho $=0,820 ; \mathrm{p}<0,01)$. Observou-se correlação significativa tanto dos escores de valência $($ rho $=0,958)$ quanto dos escores de alerta $($ rho $=0,836$; $\mathrm{p}<0,01)$ para com os dados da versão brasileira nos 20 conjuntos.

Conclusões: O material desenvolvido propicia a neurocientistas a possibilidade de manipulação e controle de variáveis relacionadas ao processamento emocional, e também pode contribuir para investigações com enfoque psiquiátrico.

Descritores: IAPS, emoção, nível de alerta, imagens, avaliação em saúde.

\begin{abstract}
Introduction: The way in which emotional stimuli are processed and relate with cognition has many implications in the mental health field. As a result, there is a need for effective instruments that allow measuring emotion.

Objectives: To create 20 sets of thematically, visually, and emotionally associated pictures using the International Affective Picture System (IAPS), and to select the most representative picture in each set; to investigate the correlation between associative factors (emotional, thematic, and visual); and to assess the emotional correlation between the 20 sets and Brazilian normative data.

Methods: A total of 353 participants evaluated the sets and defined to what degree the pictures in each set were associated with one another, and also which picture was the most representative of each set.

Results: Eighteen of the 20 sets presented high association results in the three factors assessed. Hierarchical representations were obtained for the 20 sets. There was a significant positive correlation between thematic and visual association $($ rho $=0.820 ; \mathrm{p}<0.01)$. Both valence $($ rho $=0.958)$ and arousal (rho $=0.836 ; \mathrm{p}<0.01$ ) were significantly correlated with Brazilian norms in all 20 sets.

Conclusions: The sets of associated pictures created and herein described may be useful to neuroscientists, due to the possibility of manipulating and controlling variables related to emotional processing, and may also contribute to investigations with a psychiatric focus.

Keywords: IAPS, emotion, arousal, pictures, health evaluation.
\end{abstract}

\section{Correspondência:}

Lilian Milnitsky Stein, Pontifícia Universidade Católica do Rio Grande do Sul, Pós-Graduação em Psicologia, Av. Ipiranga, 6681, prédio 11, sala 940, CEP 90619-900, Porto Alegre, RS, Brasil. Tel./Fax: (51) 3320.3500, ramal 7737. E-mail: lilian@pucrs.br

Não foram declarados conflitos de interesse associados à publicação deste artigo.

Copyright (C) Revista de Psiquiatria do Rio Grande do Sul - APRS 
"Uma imagem vale mais do que mil palavras." Pode-se dizer que esse conhecido ditado popular está, de certo modo, fundamentado no âmbito científico pelo que se conhece por efeito de superioridade da imagem (picture superiority ef$f e c t)^{1-3}$, ou seja, a ideia de que imagens são processadas com mais rapidez e melhor lembradas do que palavras. O efeito de superioridade da imagem se daria devido a um primeiro estágio de análise visual e codificação simbólica, que, em seguida, é reforçado pelo processamento semântico e lexical. Com palavras, o processamento é essencialmente apenas semântico (esse primeiro estágio não ocorre). Logo, as imagens teriam a vantagem de, em paralelo ao processamento semântico e lexical, também terem processados os atributos físicos e perceptuais que compõem a estrutura do objeto ${ }^{4}$. Para Weldon \& Roediger ${ }^{5}$, a vantagem das imagens sobre estímulos verbais se daria porque a etapa de codificação ocorre de forma mais elaborada e profunda, por serem as imagens estímulos mais ricos, complexos e distintos.

O International Affective Picture System (IAPS) ${ }^{6,7}$, um banco normatizado de imagens que eliciam diferentes emoções, vem sendo amplamente utilizado em estudos no campo da cognição humana e das neurociências. O IAPS é constituído por cerca de 1.000 fotografias que exibem diversas cenas da vida real (paisagens, acidentes, esportes, bebês, violência, sexo) e possui normas baseadas no modelo dimensional da emoção, já reproduzidas em países como EUA ${ }^{8}$, Espanha ${ }^{9}$ e Brasil $^{10}$. Essas normas foram estabelecidas para as dimensões emocionais de valência, alerta (arousal) e dominância, valendo-se de uma avaliação subjetiva através da escala pictográfica não verbal Self-Assessment Manikin (SAM) ${ }^{11}$, de 9 pontos. As medidas obtidas através da escala SAM, em geral, apresentam alta correlação com respostas fisiológicas (como condutância elétrica da pele e batimentos cardíacos) ${ }^{12}$. Os estímulos que geram níveis baixos de valência são descritos como negativos ( 1 a 3,99 pontos), níveis médios como neutros $(4$ a 5,99$)$ e níveis altos são descritos como positivos (6 a 9). Já na dimensão alerta, os estímulos são descritos como não estimulantes ( 1 a 5,99) ou estimulantes $(6 \text { a } 9)^{13}$. A dimensão dominância segue o mesmo padrão de pontuação, porém tem sido pouco abordada nos estudos sobre emoção.

Por se tratar de material não verbal normatizado, a utilização do IAPS em pesquisas em diferentes países e culturas tem permitido a comparação dos seus resultados ${ }^{14,15}$, sem a limitação da falta de equivalência linguística típica dos materiais verbais. Além disso, outras possíveis normatizações relativas ao IAPS têm sido propostas. Uma delas buscou categorizar as fotografias distinguindo-as por emoções mais específicas, postulando que um estímulo, além de avaliado com uma valência negativa, também poderia ser discriminado entre medo, nojo, tristeza e raiva, por exemplo ${ }^{14}$. Outro estudo ${ }^{15}$ acrescentou às imagens outras seis dimensões: familiaridade, significado, distintividade, memorabilidade, surpresa e consequenciabilidade.

Variados estudos enfocando populações clínicas têm utilizado o IAPS para investigar as relações entre respostas emocionais e uma determinada condição clínica. Sendo assim, há recentes investigações sobre as influências de cunho emocional na área da dependência química ${ }^{16,17}$, esquizofrenia $^{18}$, bem como relacionadas a idosos ${ }^{19}$ e a infratores ${ }^{20}$ com $^{2}$ e sem transtornos psiquiátricos.

Embora haja uma gama variada de temáticas nas imagens do IAPS, não há uma categorização normatizada com relação a esses temas, lacuna esta que o presente estudo pretende abarcar. O estudo do efeito da associação temática entre estímulos é bastante recorrente nas pesquisas envolvendo processos cognitivos, tais como memória e linguagem. Por exemplo, o clássico procedimento conhecido como DRM (Deese-Roediger-McDermott) ${ }^{21}$ consiste na utilização de listas de palavras associadas semanticamente e é capaz de induzir altas taxas de falsas memórias ${ }^{22}$. No paradigma DRM, cada lista é constituída por 15 palavras-alvo (por exemplo, sorriso, felicidade, festa, vida, amigos, família, paz, nascimento, filhos, euforia, pular, diversão, sucesso, carnaval e gol) associadas semanticamente a uma palavra-tema (por exemplo, alegria). O procedimento padrão consiste na apresentação das palavras-alvo na fase de estudo e a inclusão da palavra-tema na fase de teste, sendo frequente o falso reconhecimento ou recordação da palavra-tema como tendo sido também apresentada na fase de estudo. Na versão brasileira ${ }^{23}$ do DRM, também foram obtidos índices de valência e alerta para essas listas.

Tendo como inspiração o modelo do procedimento DRM, foi objetivo principal deste estudo construir 20 conjuntos de imagens associadas a partir do IAPS, através da coleta de índices relativos ao grau de associação temática, visual e emocional de cada conjunto. Também foram objetivos deste estudo: eleger a foto mais representativa de seu respectivo conjunto; verificar a correlação de emocionalidade dos 20 conjuntos com os dados da versão brasileira do IAPS; investigar a correlação entre os três fatores de associação (emocional, temática e visual); e representar graficamente os conjuntos dentro do espaço afetivo.

\section{Método}

\section{Participantes}

Participaram do estudo 353 estudantes universitários e de cursos técnicos de instituições públicas e privadas dos cursos de psicologia, administração de empresas, engenharia e técnico em contabilidade/administração na cidade de Porto Alegre (RS), escolhidos por conveniência e de forma anônima e voluntária. Quanto à distribuição por sexo, 191 participantes eram mulheres $(54,1 \%)$ e 162 homens $(45,9 \%)$, todos maiores de 18 anos, com média de idade de 23,6 anos (desvio padrão, DP $=5,8$ anos). Foram realizados contatos prévios com os professores das instituições de ensino, e selecionaram-se aqueles que permitiram o uso das salas de aula e dos equipamentos de exibição para que os experimentos ocorressem em suas turmas. 


\section{Material}

A partir do banco de 1.196 fotos do IAPS, 20 conjuntos com oito fotos cada foram previamente montados com base na similaridade temática, visual e emocional das imagens, de acordo com a avaliação de sete juízes independentes (três mulheres e quatro homens de 20 a 30 anos, sendo quatro com ensino superior completo e três incompleto). A lista de imagens selecionadas é apresentada na Tabela 1.

Tabela 1 - Imagens selecionadas para compor os 20 conjuntos

\begin{tabular}{|c|c|c|c|c|c|c|c|c|}
\hline \multirow{2}{*}{$\begin{array}{l}\text { Conjuntos } \\
\text { Bebês }\end{array}$} & \multicolumn{8}{|c|}{ Código das fotos selecionadas no IAPS } \\
\hline & 2660 & 2260 & 2058; & 2050; & 2057; & 2153 & 2071; & 2070 \\
\hline Cobras & 1050 & 1052 & 1026; & 1070; & 1051 & 1113; & 1022; & 1040 \\
\hline Sexo & 4669 & 4659; & 4652 & 4680; & 4694; & 4656 & 4670; & 4695 \\
\hline Comida & 7350 & 7230 & $7351 ;$ & $7481 ;$ & 7475 & 7220 & $7289 ;$ & 7402 \\
\hline Acidentes & 9900 & 9901; & $9902 ;$ & 9903; & 9910 & 9911; & 9912; & 9920 \\
\hline Cogumelos & 5500 & 5510 & 5520 ; & 5530 & 5531 & 5532 & 5533; & 5534 \\
\hline Armas & 6250 & 6260 & 6230 & 2811; & 6443; & 6190 & 6200; & 6210 \\
\hline Pais e filhos & 2152 & 2150 & 2311; & 2165; & 2160 & 2154; & 2310; & 2540 \\
\hline Nuvens & 5551 & 5891; & 5594; & 5982 & 5870 & 5593 & 5990; & 5991 \\
\hline Faces desfiguradas & 3000 & 3168 & 3053; & 3100 & 3051 ; & 3030 & 3060; & 3101 \\
\hline Casais & 4623 & 4599; & 4640; & 4610 & 4625 & 4601 & 4609; & 4624 \\
\hline Corpos mutilados & 3063; & 3120 & 3110 & 3061; & 3130 & 3015 & 3016; & 3102 \\
\hline Violência & 6560 & 6350 & 6313; & 6360 & 6550 & 6315 & 6530; & 6540 \\
\hline Esportes radicais & 8034; & 8186; & 8200; & 8030; & 8021; & 8031; & 8192; & 8193 \\
\hline Animais ferozes & 1300 & 1114; & 1932; & 1525 & 1302 & 1301 & 1930; & 1120 \\
\hline Montanhas & 5600 & 5629 ; & $5628 ;$ & 5820 & 8191; & 5631 & 5660 & 5700 \\
\hline Esportes & 8280 & 8179; & 8090; & 8041; & 8050 & 8040 & 8130; & 8161 \\
\hline Homens & 2512 & 2210 & 2190; & 2214 & 2200 & 2215 & 2493; & 2630 \\
\hline Utensílios domésticos & 7057 & 7000 & 7233; & 7080; & 7035 & 7004 & 7006; & 7009 \\
\hline Figuras abstratas & 7182 & 7237 & 7160 & 7183; & 7238 & 7207 & 7247 & 7248 \\
\hline
\end{tabular}

IAPS $=$ International Affective Picture System.

Posteriormente, foi realizado um estudo-piloto com 12 participantes, a fim de consolidar e aperfeiçoar os conjuntos e o procedimento de coleta dos dados. Finalmente, foram criadas quatro diferentes sequências de apresentação das fotografias, alterando-se a ordem de apresentação dos conjuntos e também das fotos dentro de cada conjunto. Cada uma dessas quatro sequências de apresentação continha 11 conjuntos para serem avaliados, sendo que a primeira servia de treino aos participantes para que eventuais dúvidas fossem dirimidas.

Para avaliar a emocionalidade, a associação temática e a associação visual de cada conjunto, foi elaborado um protocolo de respostas composto pelos seguintes itens:

- Versão lápis e papel do $\mathrm{SAM}^{12}$ para avaliação de alerta e valência eliciados pelos conjuntos de fotos: escores 1 a 9, sendo 1 o nível mínimo de alerta e a valência máxima negativa e 9 o nível máximo de alerta e de valência positiva.

- Duas escalas Likert com gradação variando de 1 (nada associadas) a 5 (totalmente associadas), uma para aferir o nível de associação temática e outra para medir o nível de semelhança visual entre as fotos de cada conjunto.
- Oito quadrados em branco para que os participantes ordenassem as fotos de 1 a 8, da mais à menos representativa do conjunto.

\section{Procedimentos}

O estudo foi aprovado pelo Comitê de Ética em Pesquisa da PUCRS. Foram seguidos praticamente os mesmos procedimentos adotados nos estudos de normatização do IAPS americano $^{8}$ e brasileiro ${ }^{24}$. A coleta dos dados foi realizada em grupos com cerca de 25 participantes, ao longo de 30 a 35 minutos.

Antes de iniciar a tarefa, os participantes eram informados sobre os objetivos gerais da pesquisa, que envolvia assistir e avaliar uma série de fotografias. Era frisado que os participantes iriam assistir a conjuntos de fotos e que deveriam fazer seus julgamentos com base no conjunto das fotos, e não nas fotos individuais. Todos os participantes voluntários assinaram termo de consentimento livre e esclarecido.

O material foi apresentado com a utilização de equipamento multimídia, através do programa Microsoft PowerPoint 
for Windows, projetado em uma tela de 1,20 x 1,50 m, em salas de aula com iluminação reduzida e silêncio. Era apresentado um conjunto de oito fotos, exibidas uma de cada vez, por 3 segundos, interpoladas por 1 segundo de tela preta. Após as oito fotos serem individualmente apresentadas, os participantes eram instruídos a avaliar o conjunto, como um todo, das fotos recém-vistas quanto ao alerta e à valência, assim como o quanto as fotos eram temática e visualmente associadas entre si. Subsequentemente, as oito fotos do conjunto recém-avaliado eram expostas simultaneamente em tamanho reduzido para que os participantes as hierarquizassem em termos de representatividade, ou seja, deviam julgar qual era a foto que melhor representava o conjunto, até aquela que menos o representava, formando um ranking. Uma vez concluídas essas etapas, apresentava-se um novo conjunto de oito fotos, e assim sucessivamente. Sem considerar o conjunto de treino, cada participante avaliou 10 conjuntos diferentes de fotos.

\section{Análise de dados}

Para as análises, foi seguido o padrão utilizado por outros trabalhos na área de avaliação de dimensões no IAPS ${ }^{8,14,15,24}$, tratando-se as avaliações como medidas intervalares a partir das quais foram calculadas as médias e os desvios padrão. Como uma medida conservadora, para não utilizar suposições de distribuição normal dos dados, foram calculadas correlações de Spearman, com $\alpha \leq 0,05$.

Médias e desvios padrão de emocionalidade (valência e alerta) e do grau de associação temática e visual foram calculados para todos os 20 conjuntos de fotos. Para cada conjunto, as médias foram computadas com base em uma amostra de pelo menos 144 avaliações de participantes (máximo de 207). Excluíram-se das médias as respostas em branco ou respostas múltiplas produzidas por um mesmo participante. Uma primeira análise constatou que a utilização de quatro diferentes sequências de apresentação das fotos não gerou um efeito de ordenamento sobre os resultados, portanto esse fator não foi incluído nas análises seguintes.

\section{Resultados}

Com base nos procedimentos de análise de dados previamente apresentados, os resultados obtidos para os 20 conjuntos em termos de emocionalidade (valência e alerta) e associação temática e visual são apresentados na Tabela 2.

Tabela 2 - Médias e desvios padrão dos 20 conjuntos segundo associação temática, associação visual, alerta e valência (em forma decrescente conforme a associação temática)

\begin{tabular}{lcccc}
\hline Conjuntos & $\begin{array}{c}\text { Associação } \\
\text { temática } \\
\mathbf{1 - 5}\end{array}$ & $\begin{array}{c}\text { Associação } \\
\text { visual } \\
\mathbf{1 - 5}\end{array}$ & $\begin{array}{c}\text { Alerta } \\
\mathbf{1 - 9}\end{array}$ & $\begin{array}{c}\text { Valência } \\
\mathbf{1 - 9}\end{array}$ \\
\hline Bebês & $4,79(0,42)$ & $4,18(0,87)$ & $4,28(2,81)$ & $8,09(1,36)$ \\
Cobras & $4,78(0,48)$ & $4,30(0,83)$ & $6,54(1,88)$ & $3,36(2,12)$ \\
Sexo & $4,78(0,44)$ & $4,28(0,76)$ & $7,75(1,56)$ & $7,79(1,64)$ \\
Comida & $4,77(0,50)$ & $3,78(1,09)$ & $6,92(2,12)$ & $7,92(1,41)$ \\
Acidentes & $4,74(0,44)$ & $4,18(0,71)$ & $6,78(1,76)$ & $2,15(1,43)$ \\
Cogumelos & $4,72(0,55)$ & $4,11(0,93)$ & $4,23(1,90)$ & $5,23(1,88)$ \\
Armas & $4,63(0,66)$ & $4,08(0,87)$ & $6,82(1,74)$ & $2,68(1,75)$ \\
Pais e filhos & $4,63(0,55)$ & $4,10(0,82)$ & $3,28(2,37)$ & $8,04(1,22)$ \\
Nuvens & $4,62(0,61)$ & $4,21(0,80)$ & $3,15(2,13)$ & $7,57(1,39)$ \\
Faces desfiguradas & $4,62(0,60)$ & $4,20(0,89)$ & $7,59(1,71)$ & $1,39(0,96)$ \\
Casais & $4,56(0,63)$ & $3,95(0,95)$ & $5,36(2,49)$ & $7,58(1,43)$ \\
Corpos mutilados & $4,56(0,64)$ & $4,07(0,81)$ & $7,38(1,77)$ & $1,44(0,93)$ \\
Violência & $4,54(0,60)$ & $4,00(0,85)$ & $7,12(1,55)$ & $1,86(1,28)$ \\
Esportes radicais & $4,38(0,72)$ & $3,79(0,91)$ & $6,41(2,30)$ & $7,38(1,55)$ \\
Animais ferozes & $4,32(0,72)$ & $3,68(0,99)$ & $6,82(2,78)$ & $2,78(1,71)$ \\
Montanhas & $4,27(0,84)$ & $3,93(0,80)$ & $4,81(2,38)$ & $7,48(1,67)$ \\
Esportes & $4,27(0,81)$ & $3,32(1,08)$ & $6,19(2,34)$ & $7,29(1,56)$ \\
Homens & $4,11(0,93)$ & $3,50(1,10)$ & $4,16(1,74)$ & $4,92(1,61)$ \\
Utensílios domésticos & $3,65(1,00)$ & $2,83(1,18)$ & $3,68(2,09)$ & $5,91(1,59)$ \\
Figuras abstratas & $3,28(1,00)$ & $2,81(1,09)$ & $5,07(1,84)$ & $5,00(1,68)$ \\
\hline Assocaço ten & & &
\end{tabular}

Associação temática e visual: gradação de 1 a 5; alerta e valência: gradação de 1 a 9; desvios padrão entre parênteses. 
Em termos de associação temática, observa-se que 18 dos 20 conjuntos (90\%) mostraram escores médios acima de 4 pontos, medida que indica que foram avaliados como contendo fotos muito associadas entre si. Considerando que a escala varia de 1 a 5 pontos, os escores médios de associação temática foram de 3,28 ( $\mathrm{DP}=1,00)$ para o conjunto menos associado (figuras abstratas) e de $4,79(\mathrm{DP}=0,42)$ para o mais associado (bebês).

Com relação à associação visual, o intervalo dos escores médios foi de 2,81 (DP = 1,08), para figuras abstratas, a 4,30 (DP $=0,82$ ), para cobras. Constatou-se que 12 conjuntos foram avaliados como muito associados visualmente, ao passo que oito tiveram seus escores inferiores a 4 pontos; apenas dois conjuntos apresentaram escores abaixo de 3 pontos (figuras abstratas e utensílios domésticos).

Em todos os conjuntos, elegeu-se uma fotografia como sendo a que melhor representava o conjunto das oito fotos. Para essa escolha, o critério adotado foi o da maior frequência com que uma foto foi colocada na primeira posição da hierarquia de representatividade (ou seja, a mais típica de cada conjunto de fotos)*.

Para avaliar a existência de uma relação entre os fatores associação temática, associação visual, valência e alerta, foi calculada a correlação de Spearman. A análise mostrou uma forte correlação positiva entre os fatores associação temática e associação visual (rho $=0,820 ; p<0,01$ ), enquanto que, entre os outros fatores, não foram observadas correlações significativas (nível de significância estatística, $\mathrm{p}_{\mathrm{s}}>0,05$ ).

Foi investigada também a presença de correlações entre as avaliações de emocionalidade dos 20 conjuntos e as avaliações obtidas na versão brasileira do IAPS ${ }^{24}$. Para isso, foram colhidos os escores de alerta e valência da versão brasileira de cada uma das fotos utilizadas no presente estudo. A partir desses escores, foram computadas as médias de alerta e valência para cada conjunto de oito fotos. Então, realizou-se uma correlação entre essas médias encontradas (de alerta e valência) para cada um dos 20 conjuntos e as médias obtidas no presente estudo. Foi observada uma forte correlação positiva tanto dos escores de valência (rho = $0,958)$ quanto dos escores de alerta ( $\left.r h o=0,836 ; \mathrm{p}_{\mathrm{s}}<0,001\right)$ para com os dados da versão brasileira, ou seja, as médias de valência e alerta encontradas para os 20 conjuntos neste estudo correlacionaram-se positivamente com as médias dos escores individuais de cada foto obtidas na versão brasileira do IAPS.

Os estados afetivos eliciados pelos 20 conjuntos de fotos podem ser representados graficamente por sua localização em um espaço bidimensional denominado "espaço afetivo" 25 . Esse espaço é constituído pelas dimensões valência (ordenada) e alerta (abscissa). A Figura 1 mostra que a distribuição dos 20 conjuntos dentro do espaço afetivo segue a mesma tendência observada nos estudos do IAPS brasileiro ${ }^{24}$ e americano ${ }^{9}$. Nenhum dos 20 conjuntos se posicionou no quadrante que indica valência negativa e baixo alerta.

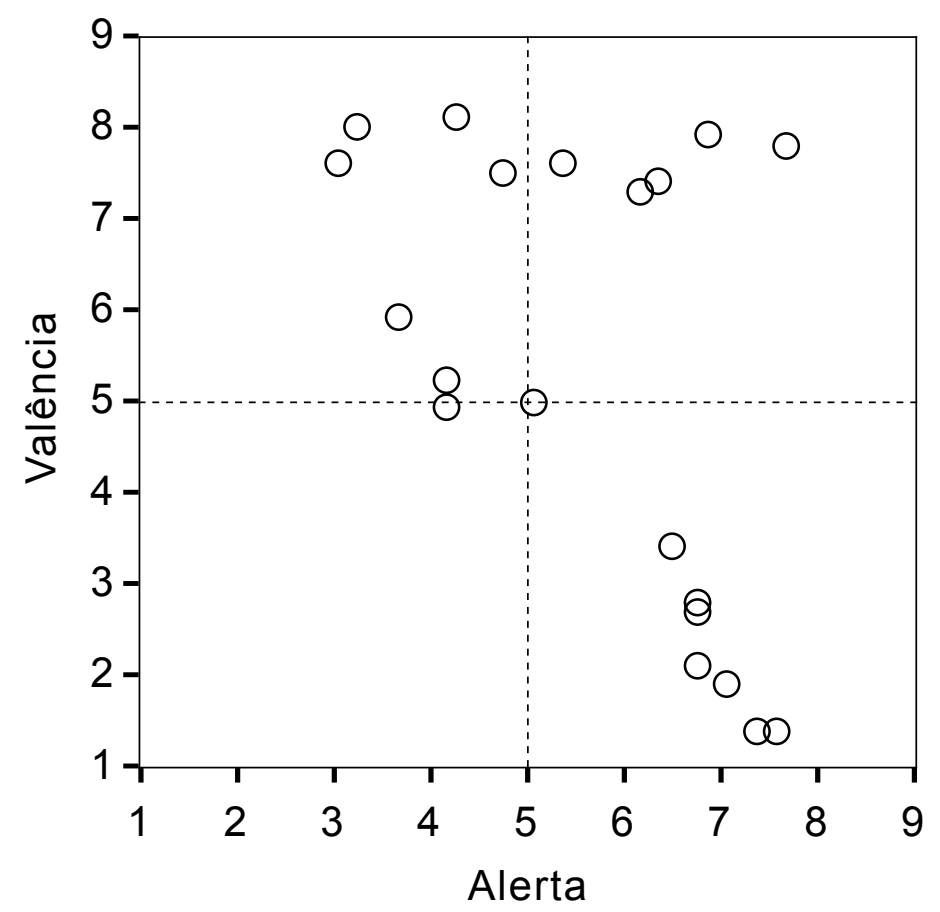

Figura 1 - Distribuição das médias de valência e alerta dos 20 conjuntos avaliados

\footnotetext{
* Os autores podem disponibilizar para consulta todos os ordenamentos hierarquizados por representatividade de cada conjunto, bem como os bancos de dados contendo os valores de associação temática, associação visual, valência e alerta dos 20 conjuntos de fotos estudados.
} 


\section{Discussão}

A proposta do presente estudo foi criar conjuntos associados de imagens a partir do IAPS, valendo-se de parâmetros de associação temática, visual e emocional. A forte correlação entre os escores de alerta e valência do conjunto das oito fotos de cada categoria e os escores obtidos pelas médias individuais de cada foto nos estudos anteriores ${ }^{9,24}$ parece indicar que há uniformidade e coesão nos conjuntos em relação à emocionalidade. No que diz respeito à associação temática, de acordo com os índices coletados neste estudo, nota-se uma tendência de forte homogeneidade interna em 18 dos 20 conjuntos. Já em relação à associação visual, a maioria dos conjuntos também se mostrou homogênea, porém em quantidade e grau um pouco menor do que na avaliação da associação temática. Para explicar essa pequena diferença, pode-se inferir que a análise visual é mais específica, direcionada a atributos concretos da imagem, o que possibilita mais pontos de discriminação. Por outro lado, a avaliação da temática se embasa mais no significado do tema representado pelo conjunto das fotos (por exemplo, cobras), de forma menos delimitada e mais inespecífica, o que favorece a conjunção e dificulta a distinção entre as fotos.

Uma hipótese para o fato de os conjuntos de figuras abstratas e utensílios domésticos terem obtido os mais baixos índices de associação temática e visual poderia ser a neutralidade emocional desses conjuntos. Estímulos com valência e alerta neutros parecem chamar e manter menos a atenção do que estímulos emocionais ${ }^{19,20,26}$. Assim, os participantes podem ter despendido menos recursos cognitivos para perceber os aspectos em comum das fotos desses conjuntos. Pode-se também supor que o conjunto de figuras abstratas obteve o menor grau de associação temática por não receber o auxílio do processamento semântico e lexical, uma vez que suas imagens não possuem uma estrutura reconhecível ou nomeável. Além disso, nesse conjunto, as próprias formas e cores também são, em sua maioria, significativamente diferentes de uma foto para outra. Por outro lado, a associação visual mais baixa em utensílios domésticos pode ter ocorrido por se tratar de um conjunto de conteúdo mais bem definido, favorecendo a observação da distintividade de cada objeto. Esses dois exemplos parecem indicar que os participantes compreenderam adequadamente a tarefa de avaliar a associação dos conjuntos de imagens.

Para aumentar a homogeneidade interna e também a quantidade de conjuntos, sugere-se que novas fotos sejam produzidas e incorporadas em estudos futuros. $\mathrm{O}$ número de oito fotos por conjunto neste estudo foi definida por uma questão de padronização e limitação relacionada à disponibilidade de fotos passíveis de formação de conjuntos temáticos distintos. Outro ponto a ser levado em conta em estudos futuros que visem aperfeiçoar a padronização deste instrumento diz respeito à luminosidade das fotos de um mesmo conjunto, uma vez que, além da emocionalidade, o nível de luz também parece influenciar o processamento das fotos do IAPS $^{26}$.

Tendo em vista o potencial de utilização desse material em diferentes campos de estudo, sugere-se que, em avaliações futuras, o instrumento seja não somente ampliado, mas também que se incluam outros grupos amostrais, tais como idosos, adultos de diferentes escolaridades e pacientes com diferentes transtornos psiquiátricos e neurológicos (por exemplo, disfunção ou lesão pré-frontal ou cerebelar). Também se sugere verificar a possível distinção nas avaliações feitas por homens e mulheres, uma vez que o sexo parece influenciar o modo como são processados estímulos emocionais ${ }^{27}$. Nesse sentido, a utilização de uma amostra composta somente por participantes com alta escolaridade neste estudo é indicada como uma limitação.

Visto que a emoção parece ser a chave que liga estados mentais e doenças físicas ${ }^{28}$, os dados disponibilizados neste estudo permitem que este material seja utilizado na interface entre emoção, cognição e comportamento. Reconhecendose que imagens são estímulos com um processamento mais facilitado do que palavras, os conjuntos do IAPS fornecem um avanço em direção a estudos mais naturalísticos que envolvam emoção, entretanto sem a perda do necessário rigor do controle experimental. 


\section{Referências}

1. Nelson DL. Remembering pictures and words: appearance, significance and name. In: Cermak LS, Craik FI, eds. Levels of processing in human memory. Hillsdale: Erlbaum; 1979. p. 45-76.

2. Paivio A. Imagery and deep structure in the recall of English nominalizations. J Verb Learn Verb Behav. 1971;10:1-12.

3. Paivio A, Csapo K. Picture superiority in free recall: imagery or dual coding? Cogn Psychol. 1973;5:176-206.

4. Snodgrass JG, McCullough B. The role of visual similarity in picture categorization. J Exp Psychol Learn Mem Cogn. 1986;12:147-54.

5. Weldon MS, Roediger HL. Altering retrieval demands reverses the picture superiority effect. Mem Cognit. 1987;15(4):269-80.

6. Center for the Study of Emotion and Attention (CSEA-NIMH). The International Affective Picture System [photographic slides]. Gainesville: University of Florida; 1995.

7. Lang PJ, Bradley MM, Cuthbert BN. International Affective Picture System (IAPS): technical manual and affective ratings. Gainesville: NIMH Center for the Study of Emotion and Attention/University of Florida; 1999.

8. Lang PJ, Bradley MM, Cuthbert BN. International Affective Picture System (IAPS): affective ratings of pictures and instruction manual. Technical Report A-6. Gainesville: University of Florida; 2005.

9. Moltó J, Montañés S, Poy R, Segarra P, Pastor MC, Tormo MP. Un nuevo método para el estudio experimental de las emociones: el International Affective Photograph System (IAPS). Adaptación española. Rev Psicol Gen Apl 1999;52:55-87.

10. Lasaitis C, Ribeiro RL, Freire MV, Bueno O. Atualização das normas brasileiras para o International Affective Picture System (IAPS). Rev Psiquiatr Rio Gd Sul. 2008;30(3):230-5.

11. Lang PJ. Behavioral treatment and bio-behavioral assessment: computer applications. In: Sidowski JB, Johnson JH, Williams TA, eds. Technology in mental health and delivery systems. Norwood: Ablex; 1980. p. 119-37.

12. Bradley MM, Lang PJ. Measuring emotion: the Self Assessment Manikin and the semantic differential. J Behav Ther Exp Psychiatry. 1994;25:49-59.

13. Kensinger EA, Corkin S. Two routes to emotional memory: distinct neural processe for valence and arousal. Proc Natl Acad Sci U S A. 2004;101(9):3310-5.

14. Mikels JA, Fredrickson BL, Larkin GR, Lindenberg CM, Maglio SJ, ReuterLorenz PA. Emotional category data on images from the International Affective Picture System. Behav Res Methods. 2005;37(4):626-30.
15. Libukman TM, Otani H, Kern R, Viger SG, Novak N. Multidimensional normative ratings for the International Affective Picture System. Behav Res Methods. 2007;39(2):326-34.

16. Arcos FA, Verdejo-García A, Ceverino A, Montañez-Pareja M, LópezJuárez E, Sánchez-Barrera M, et al. Dysregulation of emotional response in current and abstinent heroin users: negative heightening and positive blunting. Psychopharmacology. 2008;198:159-66.

17. Gilman JM, Hommer DW. Modulation of brain response to emotional images by alcohol cues in alcohol-dependent patients. Addict Biol. 2008;13:423-34.

18. Neumann A, Blairy S, Lecompte D, Philippot P. Specificity deficit in the recollection of emotional memories in schizophrenia. Conscious Cogn. 2007;16:469-84

19. Porto WG, Bertolucci P, Ribeiro RL, Bueno OFA. Um estudo dos relatos afetivos subjetivos a estímulos do International Affective Picture System em uma amostra geriátrica brasileira. Rev Psiquiatr Rio Gd Sul. 2008;30(2):131-8.

20. Wahlund K, Sorman K, Gavazzeni J, Fischer H, Kristiansson M. Attenuated subjective ratings and skin conductance responses to neutral and negative pictures in non-psychopathic mentally disordered offenders with various diagnoses. Psychiatry Res. 2010;180(1):30-4.

21. Roediger HL, McDermott KB. Creating false memories: remembering words not presented in lists. J Exp Psychol Learn Mem Cogn. 1995;21(4):803-14.

22. Brust PG, Neufeld CB, Ávila LM, Williams AV, Stein LM. Procedimentos experimentais na investigação das falsas memórias. In: Stein LM, org. Falsas memórias: fundamentos científicos e suas aplicações clínicas e jurídicas. Porto Alegre: Artmed; 2010. p. 42-68.

23. Stein LM, Feix LF, Rohenkohl G. Avanços metodológicos no estudo das falsas memórias: construção e normatização do procedimento de palavras associadas. Psicol Reflex Crit. 2006;19(2):166-76.

24. Ribeiro R, Pompéia S, Bueno O. Comparison of Brazilian and American norms for the International Affective Picture System (IAPS). Rev Bras Psiquiatr. 2005;27(3):208-15.

25. Russell JA. A circumplex model of affect. J Pers Soc Psychol. 1980;39(6):1161-78.

26. Güntekin B, Basar E. Event-related beta oscillations are affected by emotional eliciting stimuli. Neurosci Lett. 2010;483(3):173-8.

27. Lithari C, Frantzidis CA, Papadelis C, Vivas AB, Klados MA, Kourtidou-Papadel $\mathrm{C}$, et al. Are females more responsive to emotional stimuli? A neurophysiological study across arousal and valence dimensions. Brain Topogr. 2010;23:27-40.

28. D'Hondt F, Lassonde M, Collignon O, Dubarry AS, Robert M, Rigoulot S, et al. Early brain-body impact of emotional arousal. Front Hum Neurosci. 2010;4(33):1-10. 